\title{
KEINER DARF GEWINNEN - \\ Potenziale einer effektiven Medienkritik NeUer TV-CASTINGSHOWS
}

\author{
Christoph Jacke
}

»Wer singen kann, kommt rein! Sie nicht!«

(Jurymitglied zu Donald Duck 2004) ${ }^{1}$

\section{Einleitung: Protest und Kritik in den Medien}

»[D]er Sender selbst macht nie einen Fehler, eventuelle Fehler eines Programms sind immer Fehler der Programmgestaltung, Programmgestaltungsfehler sind einzig Fehler des Programmbenutzers, der Programmbenutzer stellt sich immer das Programm zusammen, das ihm gerade entspricht « (Neumeister 2002: 15).

Der Schriftsteller und DJ Andreas Neumeister bringt in diesem Zitatschnipsel seiner Sprachcollage Angela Davis löscht ihre Website den vermeintlichen Kreis der Vorwürfe gegenüber den Medien und die Gegenargumentation amüsant auf den Punkt. Betrachten wir das Aufkommen neuer Fernsehformate wie etwa Big Brother, Hilfe, ich bin ein Star, Popstars oder Deutschland sucht den Superstar (DSDS), so fällt einem vonseiten der Zuschauer die oftmalige Abneigung gegen das Format Doku-Soap im Generellen bei gleichzeitiger Anerkennung einzelner Sendungen oder Folgen auf; ein Phänomen, dass im Bereich der Werbeforschung bereits bekannt und belegt ist (vgl. Jacke/Zurstiege 2005). Vonseiten der Fernsehproduzenten - und auch hier argumentieren Werbeproduzenten ganz ähnlich - wird dem meist das Argument der Quote und des experimentellen Charakters solcher neuen Formate entgegen gesetzt. Besonders berücksichtigt werden soll im vorliegenden Beitrag die Sendung DSDS, weil diese neben einer großen medialen

1 In: Walt Disneys Lustiges Taschenbuch. Nr. 330: Der Supersänger. Berlin: Egmont Ehapa 2004, S. 6. 
und ökonomischen Aufmerksamkeit einige für den Medienwandel charakteristische Merkmale aufweist: die Kommerzialisierung der Produktion von Medienpersönlichkeiten, die Simulation von Wettbewerb, Startum und Partizipation der Rezipienten, die erfolgreiche Banalisierung der Fernsehinhalte und vor allem die Eigenschaft des Mediensystems, sich zunehmend auf sich selbst zu beziehen.

Wie aber gehen Wissenschaftler - und hier insbesondere die geforderten Kommunikations- und Medienwissenschaftler - mit diesen Formaten und ihren Protagonisten um? Zum einen gibt es die Gruppe der Format-Apokalyptiker, die mit neuen Formaten ebenso wie mit neuen Technologien das Ende der alten - und damit verbunden das Ende von Gewohnheiten - herannahen sehen. Zum anderen finden sich oft Format-Euphoriker ein, die den neuen Formaten (sowie den Technologien) besondere Möglichkeiten wie Interaktivität durch Mitspielen oder Demokratisierung von Prominenz durch das Etablieren des Jedermanns auf dem Bildschirm heraufbeschwören.

Diese Jedermanns wurden einst präproduktiv gecastet und überprüft, bevor sie überhaupt in eine Sendung gelangten, mittlerweile wird aber auch die Genese solcher potenziellen Wegwerf- bzw. Einweg-Stars medialisiert und kommerzialisiert. Bewertet werden die Alexanders und Zlatkos meist zunächst von Redaktionen und Jurys und dann vom Fernseh-Volk, von ihren Nachbarn, die es (noch) nicht geschafft haben. Gerne wird sich über deren schauspielerische und musikalische Qualitäten mokiert. Doch wird dabei verkannt, dass diese Medienpersonen Aufmerksamkeit und also Quote generieren und nicht gut singen oder schauspielern sollen. Und in dieser Funktion sind sie - zumindest kurzfristig - für das Mediensystem sehr erfolgreich (vgl. Jacke 2001).

Wer aber beobachtet und bewertet eigentlich die erwähnten Bewerter? Wer beurteilt jenseits von neunmalklugen Stammtischen und überheblichen Feuilletons diese Wettbewerbe um Quote? Die Lage der Medienkritik ist derzeit insbesondere auf dem Feld medienkulturkritischer Theorie- und Grundlagenforschung äußerst unübersichtlich und unbefriedigend. Ich möchte in meinem Beitrag eine Art Werkstattbericht aus den Forschungen zur Medienkritik jenseits von Meistererzählungen und also auch Meisterkritiken geben und am Beispiel DSDS diskutieren, um auf die Probleme der heutigen Medienkritik aufmerksam zu machen. ${ }^{2}$

2 Hierbei beziehe ich mich auf die Resultate meiner ausführlichen Studie verschiedener Medienkulturtheorien in Jacke 2004. 


\section{Protest und Kritik in Medienkulturtheorien}

Betrachtet man Medienkritikpotenziale in Medienkulturtheorien wissenschaftlicher Prägung, die sich mit populären Phänomenen der Medien beschäftigen, so fallen zunächst drei Stränge ins Auge, die aus ganz unterschiedlichen Perspektiven und mit ebensolchen Resultaten argumentieren und hier auf das Format DSDS angewendet werden sollen:

- Kritische Theorie(n),

- Cultural Studies,

- Medienkulturwissenschaft(en).

Alle drei weit gefächerten Felder berücksichtigen Popkultur und deren Phänomene im Rahmen mediengeschichtlicher Entwicklungen, beurteilen diese aber sehr unterschiedlich. Das oft erscheinende ausdrückliche Bewerten und Vorverurteilen neuer Medienphänomene in der Mediengeschichte birgt eine gewisse Gefahr, die der Weimarer Medienphilosoph Joseph Vogl jüngst folgendermaßen pointierte:

»Eine Geschichte der Medienkritik scheint nicht nur älter und geläufiger zu sein als eine Historiographie der Medien selbst. Medienkritik und Medienfunktion sind vielmehr kaum voneinander $\mathrm{zu}$ trennen: als ob sich die Wirkung von Medien nur mit der Kritik dieser Wirkung verwirklichen dürfte. Medienkritik ist selbst ein Medieneffekt«(Vogl 2002: 134).

Diese Medieneffektivität von Medienkritik zeigt sich insbesondere bei neueren Fernsehformaten. Die Fernsehproduzenten selbst betonen nicht ganz zu Unrecht die Unterstützung und sogar Funktionalisierung in Sachen öffentlicher Aufmerksamkeit durch etliche Kritiken in den Medien. Indem sie sich gegen vermeintlich triviale und durchkommerzialisierte Formate und deren Handlungsträger wenden, fördern die Feuilletonisten und sonstigen Kritiker zumeist doch nur das Anliegen, Öffentlichkeit herzustellen und im Anschluss daran verschiedene Arten von Teilnahmebereitschaft zu erzeugen; Möglichkeiten der effektvollen Nicht-Zustimmung innerhalb von Artikulationen bleiben keine. Die höchste Strafe bleibt das Ignorieren, welches aber eben keine Auseinandersetzung bedeutet.

Nichtsdestotrotz berücksichtigen bestimmte Ansätze von Kritik vermeintlich triviale Inhalte und Formate, während die Hauptströme ganzer Wissenschaften sie schlichtweg ignorierten. 


\subsection{Kritische Theorien: Die Große Weigerung}

»Für mich ist die Welt nicht mehr in Ordnung. Nicht früh um Sieben und auch nicht nach der Tagesschau. Für mich heißt das Wort zum Sonntag 'Scheiße und das Wort zum Montag >Mach mal blau« (Ton Steine Scherben: »Wir müssen hier raus!«, 1972).

Sicherlich sahen sich die -klassischen Vertreter Kritischer Theorie nicht als puren Medieneffekt. Dass sie für große Kreise von Lesern aber durchaus eine über Medien vermittelte Faszination ausübten, dürfte als unbestritten gelten. Und auch wenn Ihnen - wie zuletzt von Norbert Bolz (2004) - der Tod bescheinigt wird, so entwickelt sich m. E. gerade in Zeiten der zunehmenden Beschleunigung, Kontingenz, Komplexität und Reflexivität von Medienangeboten ein vorsichtiges Wiederentdecken und Aktualisierungen kritisch-theoretischer Überlegungen (vgl. etwa Behrens 1999, 2003, 2004), sicherlich auch, weil diese sich bemühen, Ordnung in postmoderne Unübersichtlichkeiten zu bringen.

Was kann aus Betrachtungen zur Kritischen Theorie klassischer und fortgeschrieber moderner Prägung für eine Analyse popkultureller Phänomene wie DSDS geschlossen werden? Und wo gibt es Anschlussmöglichkeiten an andere, neue(re) theoretische Überlegungen? Kurzum: Worin besteht der Orientierungswert kritischer Gesellschaftstheorie für gegenwärtige Problemlagen? (vgl. dazu ausführlich Jacke 2004: 30-159).

Anhand der Werkzeugkisten Kritischer Theorie lassen sich auch weiterhin Zusammenhänge von Kunst, Kultur, Medien und Gesellschaft und vor allem historische Dynamiken des Zivilisationsprozesses und somit fortgeschrittenen gesellschaftlichen Wandels analysieren und kritisieren. Dabei müssen allerdings insbesondere die klassischen Betrachtungen der wachsenden Bedeutung der Massenmedien für diesen Wandel berücksichtigt werden. Ein verschärfter wissenschaftlicher Blick auf diese Bereiche sollte medienkulturwissenschaftlich und kommunikationswissenschaftlich geschult sein, und genau dies ist bei den meisten hier erwähnten Autoren nicht der Fall sei es durch deren Eingebundenheit in eine Zeit, als die Rolle der Massenmedien eher für kriegerische Propaganda denn für Alltagsgestaltung diskutiert wurde, oder sei es, weil sie den Medien auf dem freien Markt sehr skeptisch gegenüber stehen oder sie sogar nicht weiter in Erwägung ziehen. ${ }^{3}$

3 Göttlich (2003: 47) hingegen schließt in seine Definition des Begriffs Kulturindustrie, speziell seit den 1960er Jahren, die massenmediale Vermittlung zwingend mit ein. Dies erscheint aus medienkultur- und kommunikationswissenschaftlicher Perspektive dringend erforderlich, während philosophische Be- 
Hieran wird klar, warum die kulturkritische >Keule< aus gesellschaftstheoretischer Sicht gegen einzelne Fernsehformate oder Protagonisten wie im Fall von DSDS nicht nur übertrieben erscheint, sondern nicht funktioniert.

Versucht man aber von Einzelfällen auf die Mediengesellschaft zu abstrahieren, bleibt unweigerlich die Kulturindustriethese mitsamt ihrer Fortschreibungsversuche als Kern kritisch-theoretischer Beobachtungssettings aktuell. Die Permanenz einer kritischen Selbst-Reflexion ist der Form der negativen Dialektik in dieser These eingeschrieben. Auch wenn viele der Formulierungen der klassischen Kritischen Theorie dementsprechend außerordentlich negativ und teilweise sogar aussichtslos anmuten, so haben die Denker doch in ihrer eigenen Entwicklung (Adorno) sowie in ihrer Abfolge (Adorno $\rightarrow$ Horkheimer $\rightarrow$ Löwenthal $\rightarrow$ Marcuse $\rightarrow$ Benjamin) diese Totalität aufgebrochen oder zumindest aufgeweicht. Dies geschah interessanterweise eher in Bezug auf kommunikations- und medienwissenschaftliche Aspekte wie die abnehmende Stärke des Manipulationsverdachts seitens der Medien, Wirkungsannahmen und Machtzuschreibungen bzw. den Einbezug unterschiedlicher Lesarten seitens des Rezipienten.

Aus der klassischen Generation Kritischer Theorie (v.a. Horkheimer, Adorno, Löwenthal, Marcuse, Benjamin) ist nicht der einheitliche Ansatz zu isolieren. Entweder verbleibt man in starren Gerüsten, katalogisiert Kulturen dementsprechend und kritisiert Medienpersönlichkeiten, Fernsehformate und das Fernsehen im Ganzen als falsch, verdummt und verdummend: Somit wären etwa die Protagonisten von DSDS nichts als Reklame für sich selbst, den Sender und die Musikindustrie, Pseudo-Kunst für die Selbstverblendung der Zuschauer, die wiederum über die Massenmedien lancierte Parolen unkritisch aufgreifen (vgl. Horkheimer/Adorno 2000: 128-176, Adorno 1992: 167-184, Adorno 2003: 69-80). Oder aber man versucht, wie Habermas (1990, 1995a, 1995b), Prokop (1974, 2003) und Behrens (1999, 2003, 2004) es ansatzweise in der Folge exerzierten, das Progressive in der Massenkultur zu finden.

Aus dem Konglomerat der hier erwähnten Autoren >klassischer Kritischer Theorie< lassen sich einige zentrale Thesen schließen, die es bei den modernen Fortführungen auch auf ihre Anwendbarkeit auf neue Fernsehformate wie DSDS zu überprüfen gilt:

- Verschiedene Ebenen von Kultur sind beobachtbar und bewertbar.

- Durch eine Dialektik geraten diese Ebenen in einen Wandel, der aber nicht zwangsläufig zum Fortschritt führt.

trachtungen gerne darauf verweisen, dass Kulturindustrie eben nicht nur aus Massenmedien bestehe, sondern im Sinne Adornos die herrschende Form der geistigen Produktion bedeute (vgl. Paetzel 2001: 91). 
- Die beiden Ebenen sind in Main und Sub einteilbar: Innerhalb der klassischen Kritischen Theorie wurde lediglich durch eine analoge Bewertung der Ebenen für die Gesellschaft in Kunst (high) und Massenkultur (low) kategorisiert. Nimmt man die Bewertungen heraus und entdramatisiert die Beobachtung, so gelangt man an den Punkt, dass Kultur von der Industrie für die Masse (Main) und von der Kunst oder anderen nichtindustriellen Gruppen für interessierte Gruppen in Form von Spezialisten (Sub) abläuft.

- Durch einen Perspektivenwechsel haben die Denker der klassischen Kritischen Theorie zunächst überhaupt erst einmal ihre Blicke auf das Main geworfen, welches zuvor schlichtweg ignoriert worden war. ${ }^{4}$

- Schließlich wurde sogar versucht, erste Anzeichen einer Emanzipation des Main als auch der Möglichkeiten des Sub zu beobachten.

- Die Möglichkeiten des Sub ähneln denen der Kunst als gesellschaftlicher Kritik: Nicht von außerhalb können sie genutzt werden, sondern nur aus dem System, aus der Gesellschaft heraus. Der verdinglichten Welt soll gewissermaßen der Spiegel vorgehalten werden. Allerdings sollte die Mimesis nicht perfekt sein, denn dann stünde die Formauflösung der Form nicht mehr gegenüber. Und diese Form muss doch auch befremden, um etwas Neues zu schaffen, muss autonom vom Alltäglichen sein und doch auch in es hineinragen.

- Neben kultureller Produktion und kulturellem Werk (Adorno) muss auch die Rezeption kultureller Angebote mehr beachtet werden (Benjamin).

Bei den erwähnten Fortführungen von Habermas, Prokop und Behrens ließe sich dann - auf die oben genannten Thesen bzw. deren Aktualisierung bezogen - feststellen:

- Es muss nicht zwingend die Unwahrheit der Gesellschaft als ganzer dechiffriert werden. Auch in Teilen und Fragmenten kann Kritik geübt werden und zwar von außerhalb oder innerhalb der Main-Ebene.

- Entgegen der Annahmen vor allem von Horkheimer und Adorno gibt es auf der Ebene der Massenkultur bzw. Kulturindustrie (Main) eben doch Widersprüche und Kritikmomente zu beobachten, die einer latenten Legitimation bestehender Verhältnisse entgegen wirken.

- Für eine Kritik der Gesellschaft spielen die Massenmedien eine entscheidende Rolle - ob als Chance oder Gefahr, wird unterschiedlich beurteilt.

4 Eine der frühen multiperspektivischen Betrachtungen der populären bzw. Massenkultur war der von Rosenberg/White (1957) herausgegebene Sammelband, in dem sich neben kulturkritischen Klassikern wie Adorno, Anders oder Löwenthal auch frühe Medientheoretiker wie McLuhan und Fiedler finden. 
- Mit einer Ausdifferenzierung der Kultur-Ebenen in Main und Sub sollte eine Ausdifferenzierung der Bewertungskriterien (high und low auf beiden Ebenen) vollzogen werden (Mehrdimensionalität). ${ }^{5}$

- Die Kultur-Ebenen Main und Sub sind gesellschaftsübergreifend und funktionieren nicht mehr schichtspezifisch, während die klassische Kritische Theorie Gesellschaft in Schichten unterteilt und anspruchsvollen Jazz als Populärkultur der Oberschicht beschrieb. ${ }^{6}$

- Es besteht ein wechselseitiges Verhältnis der Beeinflussung zwischen Massenkulturproduktion und -rezeption.

- Eine kritische Medientheorie muss historisch ausgerichtet sein und gesellschaftliche Übergänge - etwa zur massenmedial vermittelten veröffentlichten Meinung - deutlicher integrieren (Habermas, Prokop).

- Die klassische Dialektik der Aufklärung kann zu einer neuen Dialektik der Kulturindustrie extrahiert werden (Prokop) und die erwähnte Mehrdimensionalität somit berücksichtigen.

Erst durch die Berücksichtigung der veränderten Kontexte lassen sich Grundannahmen der klassischen Kritischen Theorie revidieren und modernisieren, ein starres Hinwegsetzen über die medienkulturellen Wandlungen und ein dementsprechend stures Festhalten an den Originalen führt keinen Erkenntnisschritt weiter. ${ }^{7}$

Gesellschaftlicher Wandel ist am Wandel der Kultur-Ebenen und deren Manifestationen (z.B. im Stil) beobachtbar, beinhaltet aber eine mitlaufende Fähigkeit zur Umstellung seitens professionalisierter Beobachter wie Journalisten und insbesondere Wissenschaftler. Diese verschiedenen Wandlungen, die letztlich in gesamtgesellschaftlichen Veränderungen münden, werden durch divergierende Dialektiken dynamisiert. Die Dynamiken spielen sich in einer Gesellschaft wiederum innerhalb funktionaler Raster - eben der Kultur-Ebenen Main und Sub - ab. Dieses Muster bleibt bestehen, kann also durchaus weiter verwendet werden und löst sich keinesfalls auf. ${ }^{8}$ Die

5 Diese Ausdifferenzierung von Bewertungen konstatiert der Kultursemiotiker Umberto Eco (2002: 177) in Bezug auf George Orwells Roman 1984 als Vergnügen erster Art für Parteimitglieder und Vergnügen zweiter Art für Proleten.

6 Hierbei sollte bedacht werden, dass der Fernsehentertainer Harald Schmidt mit der Bezeichnung seines Ex-Senders SAT 1 als »Unterschichtenfernsehen « einen Begriff geprägt hat, der mittlerweile weit über die Feuilletons in die Gesellschaft diffundiert ist und erstaunlich Konjunktur macht. Dementsprechend wäre DSDS Unterschichtenfernsehen im Pseudo-Wettbewerbsformat, gewissermaßen low des Main.

7 Vgl. zu einer Verwertung der Überlegungen klassischer Kritischer Theorie für eine Populärkulturanalyse auch Göttlich 2003.

8 Hier widerspreche ich Kaspar Maase (2003: 52), der von einer gebrochenen Polarität zwischen Hoch- und Massenkultur redet. 
gesellschaftlichen Beobachter entdramatisieren lediglich im Zuge des gesellschaftlichen Wandels die Bewertungen der Ebenen dieses Rasters bzw. Musters, welches Kultur genannt werden kann. Neben den Bewertungen der Ebenen wandeln sich natürlich auch die Inhalte der Stufen oder wie Strinati (1995: 73) es für die Ebenen Core (Main) und Periphery (Sub) bezeichnet: Der Mechanismus bleibt derselbe, aber die Moden wechseln beständig.

Ferner ist neben der Ausdifferenzierung der Kultur-Ebenen auch eine Verfeinerung der Kritikmöglichkeiten zu beobachten: War es bei Horkheimer und Adorno die Utopie und bei Marcuse die große Weigerung, so suchen die modernen Kritischen Theoretiker Kritik- und Veränderungspotenziale in wesentlich konkreteren Phänomenen und erscheinen somit auch kompatibler für Phänomene wie DSDS. Dadurch entsteht eine Verästelung von Kritik und Innovation, die nun nicht mehr makropolitisch, sondern im alläglichen Kleinen abläuft. Es geht nicht mehr um die Verurteilung des Fernseh- und darüber gelagerten Mediensystems, es geht nicht mehr um die generellen Weisen von Produktion, Distribution, Rezeption und Weiterverarbeitung, sondern um diese Ebenen innerhalb konkreter Formate, die erst durch die Systematisierung und Konkretisierung (be-)greifbar werden.

Was also bleibt das vorläufige Ergebnis eines Re-Readings der klassischen und modernen Kritischen Theoretiker? Die mannigfaltigen Unvereinbarkeiten der Argumentationen von vor allem Horkheimer und Adorno sollten nicht nachträglich aufgelöst werden, denn sie sind nicht aufzulösen: »Hätte Adorno gefragt, um die Fragen zu beantworten, dann hätte er darauf gewiss so gut begründete Antworten geben können wie nur irgendeiner « (Brock 1989: 237).

Vielmehr gilt es, die Entwicklung von Problemstellungen und Problementdeckungszusammenhängen bei diesen Autoren genau zu beobachten, um sich deren Analyse- und Problematisierungsschärfe ansatzweise anzueignen. Anhand dieser kann es gelingen, eine aktualisierte, neue kritische Kulturund Medientheorie zu konzipieren, die allerdings zumeist auf der Makroebene der Gesellschaftstheorie verharren muss und ja auch will. Es kann ihr nicht um die ausschließliche, pauschale Abqualifizierung einzelner Medienpersonen wie Casting-Teilnehmer oder Casting-Juroren oder Rezipienten gehen $^{9}$ - das wäre zu einfach. Es muss ihr langfristig um die Veränderung der gesamten Mediengesellschaft gehen.

9 Solche Pauschalisierungen wirken oft schwerfällig und werden immer wieder empirisch widerlegt (vgl. zu Werberezipienten Jacke/Zurstiege 2005, zu den Casting-Show-Rezipienten bei DSDS die innovative Studie von Lothwesen/ Müllensiefen/Tiemann/Matterne in diesem Band, zu den Juroren-Äußerungen bei DSDS von Appen in diesem Band), aber man kann sie der Kritischen Theorie 
Die oben zusammengefassten Thesen aus klassischer als auch fortgeschriebener Kritischer Theorie gelten als Basis für viele der Startoperationen der Cultural Studies, wobei Vertreter dieser Forschungsrichtungen durchaus auf kritische Distanz sowohl zur Larmoyanz klassischer Kritischer Theorie als auch zur oftmaligen Dogmatik oder Verschlagwortung ihrer sich als kritisch verstehenden Fortschreibungen gehen, prägnant artikuliert von Paul Willis, einem ehemaligen Mitarbeiter des Birminghamer Centre for Contemporary Cultural Studies (CCCS), der Wiege der britischen Cultural Studies: »Wir können diesen idealistischen Stoßseufzern ruhig unseren Segen geben, nur glauben dürfen wir nicht an sie (Willis 1978: 44).

\subsection{Cultural Studies: Subversive Lesarten}

»Alle hatten die richtige Idee gefunden. Mit großer Freude machten sie sich auf. Und die schufen gute Instrumente. Aber wollten sie wie eine Gruppe klingen? Und sie hatten gute Transporter gebaut, aber wollten sie zusammen darauf fahren? Einige wollten einfach nur mal ihre Lieder vorspielen. Andere wollten nur mal zum eigenen Haus gebracht werden. Und so fuhren für ein Weilchen alle zusammen los. Und so zogen für ein Weilchen alle zusammen los. Und so nahmen für ein Weilchen alle zusammen Platz« (Die Goldenen Zitronen: »Ketten bilden«, 1988).

An einem Zitat der beiden australischen Cultural Studies-Forscher Kendall und Wickham lässt sich durchaus eines der Kernprobleme von Forschungen zur Medienkultur heraus lesen: »[C]ulture was always theorised in practical intellectual fields - never as an abstract problem« (Kendall/Wickham 2001: 9). Außerwissenschaftliche Beobachtungen betrifft dieser Theoriemangel sowieso, aber vor allem in den unterschiedlichen Disziplinen und noch mehr in den Studien der Cultural Studies herrscht oft eine Neigung zum ausführlich dargestellten Einzelfall, gewissermaßen zur Kasuistik, vor. Nur selten wird abstrahiert oder gar ein Theoriegebäude der Kultur zumindest angegangen. Selbiges gilt im Übrigen auch für Ansätze und Modelle zu Medien und Kommunikation, weswegen auch immer wieder auf Halls EncodingDecoding-Modell (1999b) zurückgegriffen wird. Kultur wird oftmals mit Inhalten gefüllt, die dann wiederum en detail analysiert werden. Anhand von konkreten Formationen und deren Aktanten wird Kultur erst beobachtbar, das scheint Konsens zu sein, warum also nicht von dort aus in höhere Abstraktionsebenen vorstoßen, wie dies auch Kendall und Wickham verlan-

nicht wirklich anlasten, da es ihr um die gesellschaftliche Utopie im Ganzen geht. 
gen? Warum, so soll hier entgegnet werden, wird doch eher selten versucht, nicht nur das konkrete Produkt und seine Kontexte, ${ }^{10}$ sondern auch den Prozess Kultur im Allgemeinen zu erläutern?

Vielleicht überlagern die vielen Einzelstudien und der unbedingte Wille zum gesellschaftspolitischen Einmischen hier die dringend erforderliche theoretische Sisyphusarbeit, die in Ansätzen etwa bei Grossberg oder Lull und insbesondere bei Kellner und den >Gründervätern ' Hoggart, Thompson und Williams sehr wohl vorhanden ist (vgl. dazu ausführlich Jacke 2004: 160-215). Ein Fall wie DSDS würde bei den Cultural Studies zum einen unter Aspekten des Emanzipatorischen und der Minoritäten-Politik beobachtet, also etwa im Rahmen von Fragen nach der Beteiligung und Repräsentation von Frauen oder ausländischen Mitbürgern, Emigranten etc. Zum anderen wären typische Herangehensweisen die (bspw. ethnographischen) Studien einzelner Familien in deren alltäglicher Rezeption und Nutzung von DSDS, also wie strukturiert DSDS den Alltag, wie wird die Sendung genutzt, welche Anschlusshandlungen werden dadurch ermöglicht oder eben auch nicht.

Vorläufig festzuhalten bleibt:

- In Anbindung an Überlegungen der Kritischen Theorie beobachten auch die meisten Vertreter der Cultural Studies verschiedene Ebenen von Kultur.

- In Anbindung an die Kritische Theorie wird die besondere Bedeutung des Kapitalismus und der Medien für die moderne bzw. postmoderne Gesellschaft untersucht.

- In Anbindung an die Kritische Theorie, wenn auch sicherlich ohne deren dramatischen Hintergrund, wollen Vertreter der Cultural Studies sich positionieren, einbringen und politische Arbeit leisten.

- In Anbindung an die Kritischen Theorie scheuen die Cultural Studies sich nicht, über den eigenen disziplinären Tellerrand hinauszuschauen und Forschungen als Projekte anzusehen, die annähernd umfassend lediglich durch inter- oder besser transdisziplinäre Teams geleistet werden können.

- In Anbindung an die Kritische Theorie speisen die zentralen Forscher und Forschungen zu den Zusammenhängen von Medien, Kultur und Gesellschaft ihre Analysen mit einer Mischung aus geistes- und sozialwissenschaftlichen Ansätzen.

10 Douglas Kellner etwa untersucht ausführlich und minutiös die Medienphänomene Madonna (Kellner 1995) und Michael Jordan (Kellner 2003), seine Schlussfolgerungen in Form einer Forderung nach besserer Medienkompetenzausbildung erscheinen demgegenüber etwas lapidar. 
- In Abgrenzung zu Ausführungen der Kritischen Theorie wird ein spezielles Augenmerk auf die Kultur(en) und also auf die Lebensweisen der Menschen geworfen.

- In Abgrenzung zur Kritischen Theorie wird das Alltägliche für die Forscher der Cultural Studies interessant und das Spektakuläre im Gewöhnlichen gesucht. ${ }^{11}$

- In Abgrenzung zur Kritischen Theorie geht es den Cultural Studies um eine Egalisierung der Artefakte, Produkte und Aktanten der unterschiedlichen Kultur-Ebenen als Untersuchungsgegenstand und Thema politischer Forderungen.

- In Abgrenzung zur Kritischen Theorie bemühen sich viele der Vertreter der Cultural Studies um eine Vermeidung von Bewertungen der beobachteten Untersuchungstopoi, was allerdings selbst Kellner nur bedingt gelingt und oftmals auch nicht konsequent verfolgt bzw. durch einen letztlich offensichtlich intendierten Re-Entry der Normativität gar nicht eingehalten wird.

- Zudem bemühen sich die Cultural Studies in Abgrenzung zur Kritischen Theorie zumeist um exemplarische Phänomene, die detailliert beschrieben und interpretiert werden.

- In diesem Rahmen ist auch ein Wandel von der Kritik an der Gesellschaft im Ganzen bei der Kritischen Theorie zur Beurteilung von Kritikmöglichkeiten im Einzelnen und bis hinein in opponierende Lesarten bei den Cultural Studies zu beobachten. Die große Weigerung reduziert sich demokratisierend und emanzipierend auf die vielen Verweigerungen en miniature.

Allerdings gilt es hier, zumindest grob zwei Gruppen der Cultural Studies zu unterteilen: Erstens die eher strukturalistisch inspirierten Studien, denen es noch deutlicher an der zunächst puren Dechiffrierung symbolischer Welten (zumeist im Pop und in den Medien) liegt, und zweitens die Kulturalisten, die hinter der Analyse der alltäglichen Kultur das Aufdecken von Ideen, Missständen, ungleichen Machtverhältnissen und sublimen Beeinflussungsversuchen identifizieren wollen und somit eine klare Forderung nach fun-

11 Ganz ähnlich argumentiert Eco, wenn er das Innovative im Seriellen bei Fernsehformaten analysiert und Rezipienten eine grundsätzliche Autonomie zurechnet: »Es ist evident, dass auch das banalste narrative Produkt dem Leser erlaubt, sich in autonomer Entscheidung als kritischer Leser zu konstituieren, das heißt als ein Leser, der beschließt, die Innovationsstrategien zu bewerten, so minimal sie auch sein mögen, beziehungsweise das Fehlen von Innovation zu registrieren. Aber es gibt auch serielle Werke, die einen expliziten Pakt mit dem kritischen Leser schließen und inn sozusagen herausfordern, die innovativen Kräfte des Textes freizulegen« (Eco 2002: 168). 
dierter Kritik aufstellen (vgl. ausführlich Hall 1999a). Zur zweiten genannten Gruppe ist auch Kellner zu zählen, ohne dass er sich strukturalistischen oder poststrukturalistischen Ansätzen gänzlich verschließt. Doch genügt ihm die Feststellung aktiver Rezeption in Form von Vergnügen wie etwa bei Fiske nicht, der zu den Strukturalisten unter den Cultural Studies-Denkern zu zählen ist. Sie ist Kellner zu unpolitisch:

»Neglecting political economy, celebrating the audience and the pleasures of the popular, neglecting social class and ideology, and failing to analyze or criticize the politics of cultural texts will make cultural studies merely another academic subdivision, harmless and ultimately of benefit primarily to the culture industries themselves« (Kellner 1992: 21).

Auch Kellner geht also durchaus von aktiven Rezipienten und dementsprechend deren Möglichkeiten verschiedener Lesarten von medienkulturellen Angeboten aus und sicherlich ließe sich das bei den Rezipienten von DSDS in Teilen auch feststellen, in dem man etwa die Arten der Rezeption und Nutzung von DSDS kontextualisiert und vor allem qualitativ analysiert. Doch glorifizieren Kellner zufolge Wegbereiter wie insbesondere Fiske die aktive Rezeption zu sehr und zu pauschal. Kellner verlangt eine politische Konsequenz aus seinen Beobachtungen zu medienkulturellen Chancen und Gefahren. Dies verlangt er auch von den Beobachtern auf der Meta-Ebene, denen des Journalismus und denen noch einmal darüber gelagerten der Wissenschaft. Die Vernachlässigung wissenschaftlicher Überlegungen mit klaren politischen Zielsetzungen insbesondere in Kultur- und Kommunikationswissenschaft der USA - aber eben auch westeuropäischer Länder wie der Bundesrepublik Deutschland, in deren Medienlandschaft er schon lange einen intensiven Einblick hat - spricht für Kellner (1995: 340) für die Entpolitisierung des intellektuellen Lebens. Deswegen sieht Kellner die Cultural Studies und auch ähnliche Entwicklungen im deutschsprachigen Raum als zu herkömmlichen Disziplinen quer treibendes, politisches, kritisches Projekt, in dem sich Denker formieren, die kritische Lesarten der Medienangebote lernen und lehren wollen, wie es laut Kellner (ebd.: 336) die Frankfurter Schule, die Cultural Studies und auch postmoderne Theorien bisher versäumt haben.

Leider folgen Kellners Ansprüchen auf Ansätze einer kritischen Medienpädagogik bisher zumindest keine größeren Studien, die Kultur- und Kommunikationswissenschaft noch deutlicher in Theorie und Empirie verbinden und daraus u.a. pädagogische Maßnahmen ableiten würden. Was aber mannigfaltig zu beobachten ist, ist eine Verschmelzung von kommunikationsund medienwissenschaftlichen, von eher sozial- und geisteswissenschaft- 
lichen Ansätzen zur Medienbeobachtung zweiter oder dritter Ordnung. Auch in Deutschland ko-orientieren sich immer mehr Wissenschaftler in Forschungsprojekten und an den Universitäten aneinander. ${ }^{12}$ Dazu dürften auch die Cultural Studies in ihrer höchsteigenen Form von Subs im wissenschaftlichen Main beigetragen haben: Sie haben Innovationen und Anstöße eingebracht, ihnen kann aber bis dato insbesondere in den deutschsprachigen Wissenschaften sicherlich keine Verortung im Main nachgewiesen werden. ${ }^{13}$ Ebenso haben diese Beschreibungen in diversen Fächern und vor allem in den Kultur-, Medien- und Kommunikationswissenschaften eine verschärfte Konzentration auf den Kulturbegriff und seine Bedeutungen gelegt, denn "Culture is which mediates between people and reality, turning chaos into order (Grossberg 1998: 203). Die von Lash (1996) beschriebene Reflexivität der Gesellschaft muss sinnvoller Weise in den Wissenschaften beginnen und benötigt eine durchdachte Anwendung der diesbezüglichen Theorien auf sich selbst. Damit und mit einer intensiven und vorurteilsfreien wissenschaftlichen Beobachtung vermeintlich trivialer und alltäglicher (Medien-) Phänomene haben die Cultural Studies begonnen. Mit ihrem Duktus lässt es sich entkrampft in ein Untersuchungsfeld wie DSDS gehen, konkrete Einzelfallanalysen bestreiten und insbesondere Aspekte der Reflexivität herauskristallisieren. Ihr theoretisches Rüstzeug erscheint aber noch nicht präzise und umfassend genug, um ein Medienphänomen wie DSDS im Ganzen zu beobachten und zu beurteilen.

Die Bedeutung von Kultur für die zunehmend reflexive Mediengesellschaft wird seit geraumer Zeit vom Medien- und Kommunikationswissenschaftler Siegfried J. Schmidt betont, der sich generell um reflexivitäts-, tautologie- und autologietaugliche Theorieentwürfe bemüht. ${ }^{14}$ Deswegen erscheint ein Andocken seiner Überlegungen zu Kultur, Kommunikation, Kognition und Wissenschaft und seine Einforderung einer umfassenden Medienkulturwissenschaft an die bisherigen Ausführungen im folgenden Abschnitt nahe liegend. Dabei sollen Schmidts Überlegungen und die

12 So arbeiten etwa im Münsteraner Studiengang Angewandte Kulturwissenschaften - Kultur, Kommunikation \& Management Kommunikations-, Kultur-, Medien-, Wirtschafts-, Literatur-, Sprach-, Geschichts-, Politik-, Rechtswissenschaftler, Philosophen und Soziologen gemeinsam an der Ausbildung transdisziplinär geschulter Studierender.

13 Selbst die abgeschwächten, entpolitisierten, unparteilichen Varianten (vgl. Terkessidis 2005) dürften sich noch nicht auf Folie des wissenschaftlichen Main bewegen, wenn sie auch auf bestem Wege dorthin sind.

14 Dies schließt an Überlegungen von Hans-Magnus Enzensberger an, dass Theorie selbst medienförmig im Sinne des Rückkanals werden muss und Kulturkritik notwendig Medienanalyse bedeutet (vgl. Ernst 2002: 143). 
eigenen Fortschreibungen wiederum auf deren Tauglichkeit für Beobachtungen des Phänomens DSDS zumindest ansatzweise überprüft werden.

\subsection{Medienkulturwissenschaft: Analysepräzision}

»Image is taking over the music, and that's a sin« (Miss Kittin 2005: 17).

Während sich die meisten der jugend- und popkulturellen Bewegungen der Nachkriegszeit vom Gros der Bevölkerung absetzen wollten (Sub), um u.a. zu sich selbst zu finden und das Individuelle zu betonen (die anderen/wir), starten Raver und Technoide aus dem markierten Ich (Piercing, Tattooing, Branding etc.) heraus in eine Nacht, die im möglichst großen Wir (Main) enden soll (wir/die anderen). Beide Richtungen sind mit Schmidts Begriffsapparat der »Kultur als Programm « zu deuten, welcher dazu allerdings modifiziert werden muss. ${ }^{15}$ Lange Zeit waren zumeist überzeichnete Umcodierungen der vom Main-Programm ausgehenden Unterscheidungen seitens der Anwender von Sub-Programmen z.B. in Form von Bricolage gebräuchlich. Die Techno-Bewegung ${ }^{16}$ trachtete nach der Wiederherstellung gesellschaftlicher Harmonie unter dem Deckmantel der totalen Toleranz: The Future Is Ours, We Are Family, One World - One Future, Join The Love Republic, Access Peace lauteten einige Mottos der seit 1989 stattfindenden Berliner Love Parade.

Eines wird an diesen Beispielen ganz klar: Kultur als Programm im Sinne der Interpretation kollektiven Wissens über zentrale und periphere Kategorien operiert über eine Hauptprogrammoberfläche und mannigfaltige Teilprogramme, die wiederum Main- und Subprogrammpartikel integriert halten, die Programmteile qua Anwendungen von Aktanten zwischen diesen Ebenen mäandrieren lassen. Dabei gibt es offensichtlich einen stärkeren Diffusionsschub von den Sub- zu den Mainprogrammanwendern als umgekehrt, da Subs nachdrücklich Differenzen setzen, dadurch irritieren und gegebenenfalls Wandel verursachen. Alle diese wesentlichen Aspekte des dialektischen Gegenübers von Main und Sub im Gesamtprogramm Kultur lassen sich mit der Kulturprogrammatik von Schmidt theoretisch fassen, wobei die Überlegungen zu den Kulturebenen das Konzept sinnvoll ausdifferenzieren und speziell für Phänomene der Popkultur als auch darüber hinaus anwendbar machen (vgl. grundlegend Schmidt 1999 und 2004a: 70-107 sowie

15 Programm ist hier nicht als Sendeabfolge, sondern eher als kognitive Interpretationsfolie zu verstehen.

16 Sicherlich gilt dies eher für die großen Raves und Paraden. Auch im Techno gibt es mittlerweile feinste Ausdifferenzierungen, Retro-Phänomene und sehr wohl politische Verweigerungs- bzw. Protesthaltungen. 
modifizierend Jacke 2004: 216-265). Die Vorteile der Schmidtschen Skizze und eigener Fortschreibungen sind:

- Das Konzept beinhaltet eine große Abstraktionsfähigkeit, um Kultur nicht nur als Manifestation in Form von Gegenständen, Riten, Symbolen, sondern als deren Interpretation zu verstehen (Beobachtungsvarianz statt Objektfixierung).

- Kultur wird entdramatisiert und deskriptiv eingeordnet, ohne primären Einbezug von Wertmaßstäben (Entnormativierung).

- Die verschiedenen Kulturenhauptprogramme und -ebenen erhalten eine analytische Gleichberechtigung, wobei diese in den konkreten Beobachtungen dann durchaus asymmetrisiert werden und somit weder relativistisch noch orientierungslos wirken. Daraus ergibt sich auch eine Vergleichbarkeit verschiedener Programme und Programmebenen bzw. unterschiedlicher Programme mit gemeinsamen Programmebenen (Multibzw. Transkulturalität).

- Die kulturprogrammlichen Anwendungen sind lernfähig und lernunwillig zugleich (zunächst einerlei, ob Main oder Sub, wobei die Sub-Anwender oftmals als die Aufklärer der Anwendungsveränderungen der Main-Programme handeln): In ihrer Anwendung >benutzen< und verändern sie das Programm gleichermaßen und machen durch latente Devianz den Wandel möglich.

- Kulturprogramme sichern Orientierung und Bestand von Gesellschaft. Kulturprogramme müssen "Symbole, Geschichten, Rituale, Imaginationen [und vor allem deren Interpretationen, C.J.] anbieten, die ein gemeinsames Verständnis der Situation fingieren und dadurch erst herstellen « (Wenzel 2001: 503).

- Dadurch, dass Kulturprogramme selbst unbeobachtbar sind, kann man davon sprechen, dass es Kultur nicht gibt, man sie aber laufend anwendet: Zumeist geschieht dies unbemerkt, denn das ist ihr Sinn als sozialer Kitt und Rekonstrukteur kollektiven Wissens. Will man sie explizit beobachten, so kann man das nur indirekt über die Anwender und Anwendungen.

- Somit werden die Programmanwender auf verschiedenen Ebenen in dieses Konzept von Kultur einbezogen. Sie sind das personifizierte Vis-aVis von Autonomie und Orientierung, von Kulturprogrammresultat und Kulturprogrammveränderung.

- Das Konzept verdeutlicht die Unmöglichkeit der einen einheitlichen Kultur einer Gesellschaft und erklärt komplexe, ausdifferenzierte Teilprogramme mit jeweiligen Main- und Sub-Ebenen, woraus wiederum die 
Kontingenzerfahrungen von Mediengesellschaften herleitbar sind. ${ }^{17}$ Alle diese Differenzen sind unter dem Beobachtungsraster von Main und Sub in Kulturprogrammen zu erfassen und zu erklären.

- Die Bedeutung von Medien für die Modifikation von Kulturprogrammanwendungen wird in diesem Konzept erklärt, da Medien Kognition und Kultur via Kommunikationsermöglichung koppeln.

Da Schmidts Konzept auf hohem Abstraktionsniveau angelegt ist und zunächst nicht der fallbeispielhaften Beobachtung von Einzel-Phänomenen dient, scheint es nicht weiter verwunderlich, dass er sich bisher eher peripher zu pop- und subkulturellen Phänomenen geäußert hat. ${ }^{18}$ Durch Einführung des Ansatzes von Main und Sub in Kultur wird die Ausdifferenzierungsmöglichkeit um einen Schritt verfeinert, der nicht nur Kategoriengewichtung in zentral/peripher berücksichtigt, sondern auch Differenz-Umwertung, wie dies oft im Bereich der Popkultur der Fall ist:

»Um sich mit subkulturellen Sub-Genres auszukennen, muss man eine Fülle von hochspezialisierten Habitus-Tests und -Verfeinerungen durchlaufen, die jeden Dandy grob aussehen lassen. Doch auch diese Kenntnisse und Fähigkeiten sind wesentlich davon geprägt, dass sie sich anschließen lassen müssen« (Diederichsen 1999: 278-279).

Auch die These der bescheidener werdenden Kritik lässt sich mit der vorliegenden Modifikation von Schmidts Kulturbegriff schlüssig plausibilisieren. Nach der Umwälzung der Gesellschaft bei klassischer Kritischer Theorie, der grundsätzlichen Möglichkeit von Emanzipation auf der Ebene des Main bei moderner Kritischer Theorie und den Verweigerungen in Phänomenbeispielen und deren Lesarten seitens der Cultural Studies, erklärt sich sowohl die Schwierigkeit der großen Weigerung als auch die Verästelung der Kritikmöglichkeiten mit dem Programmbegriff: Die Subs von Teilprogrammen können eben nur in diesen Teilen um Veränderung bemüht sein und beginnen auf der Mikroebene mit Umcodierungen. Mit einem kleinen Wandel dieser Art erscheint eine gesellschaftsweite Revolution nahe liegend unmöglich. Im Gegenteil: Es gibt kein Entrinnen aus der Kultur. Anschlussfähigkeit wird ja

17 Diederichsen (1999: 281) spricht ganz ähnlich von der neuen Öffentlichkeit als neuem kulturellen Gebilde, das die alten Popkulturen abgelöst hat und die verschiedenen Arten von Mainstream und Subkultur oszillieren lässt. Weinzierl (2000) entwickelt anhand zahlreicher Beispiele eine Subkulturtheorie, die von hybridisiertem Mainstream und temporären Substream-Networks (Weinzierl 2000) ausgeht, und Holert/Terkessidis (1996) berücksichtigten die Komplexität des Kulturprogramms bereits in ihren Beschreibungen eines Mainstream der Minderheiten.

18 Sehr wohl beklagt auch Schmidt jüngst (2004b: 85-94) den Mangel an Beobachtungssettings für z.B. Unterhaltung als Kulturtechnik und Medienphänomen. 
gerade anhand der Kulturprogramme auf Dauer gestellt. Diese werden wiederum durch die Aktantenanwendungen ständig bestätigt und verändert, weswegen Kultur weder >sterben< noch >zerfallen< kann, sie existiert, wie gezeigt wurde, nicht einmal. Wenn auch aus ganz anderer Startposition, so gelangt der Soziologe Richard Münch doch zu einem sehr ähnlichen Ergebnis:

»Kreativität, gemessen an Innovationen, an der Abweichung vom Etablierten ist schon deswegen nicht abgestorben, weil es dafür sogar einen expandierenden Markt gibt. [...] Die Massenkultur eröffnet der Elitenkultur neue Marktchancen und nutzt diese auch zur beständigen Innovation ihrer Massenprodukte und zur Bedienung eines sich immer mehr diversifizierenden Marktes. Mit dem Bildungsniveau des Publikums und dessen wachsendem Distinktionsbedürfnis entstehen neue Marktsegmente für innovative Kulturprodukte« (Münch 1998: 63).

Im Falle von DSDS wird so zunächst die Komplexität des Phänomens verdeutlicht und erklärt. Ferner lässt im Zuge der Ausdifferenzierungen der Mediengesellschaft durchaus ein Potenzial von Kreativität auch in solchen Phänomenen erkennen - und sei es nur, weil deren vermeintliche Uniformität und Gleichheit im Main der Fernsehformate und -inhalte an derer Stelle wieder Subs provoziert, die sich davon absetzen wollen. Der entscheidende Vorteil eines soziokulturell-konstruktivistischen Ansatzes erscheint aber in Form der Strukturierung des Untersuchungsfeldes DSDS vorzuliegen. DSDS als massenmedialer Kommunikationsprozess lässt sich einteilen in vier Stufen, die wechselseitig aneinander gekoppelt sind: Produktion, Distribution, Rezeption/Nutzung, Weiterverarbeitung.

Ebenso müssen bei der Beobachtung von DSDS als Medien-, speziell Fernsehphänomen folgenden Ebenen von Medien berücksichtigt werden, die ansonsten nur allzu gern vermengt werden und somit zur Unübersichtlichkeit beitragen: Kommunikationsmittel, Medientechnologien, sozialsystemische Organisationen, Medienangebote.

Neben der Verdeutlichung der Komplexität eines vermeintlich trivialen Medienphänomens dürfte hier klar werden, wie aufwendig Analysen des Gesamtphänomens sein müssen, will man DSDS als Ganzes kritisieren und wie präzise sie werden müssen, will man einzelne Aspekte oder Prozesse begutachten. 


\section{Fazit: Kritikpotenziale einer zukünftigen Medienkulturwissenschaft}

Die vorübergehende und noch andauernde Bescheidenheit der (deutschsprachigen) wissenschaftlichen Medienkritik dürfte im Verlauf des Beitrags und am Beispiel DSDS deutlich geworden sein. ${ }^{19}$ Ebenso klar sind die gesellschaftlichen Rufe nach einer solchen, neuen Kritik immer lauter zu vernehmen.

Wie im Titel dieses Beitrag bereits geschrieben: Keiner darf gewinnen. Dies ist nur sekundär eine Anspielung auf adorneske Argumentationsfiguren, die eben in der Massenkultur nur anti-utopische Verlierer konstatiert hatte. Primär verstehe ich den Hinweis aber derart, dass sich eine neue Medienkritik aus Perspektive einer Medienkulturwissenschaft, die etwa im Fall von DSDS auf die Hilfe der Musikwissenschaften vor allem im Bereich der Popmusik angewiesen ist, aller Ebenen des massenmedialen Kommunikationsprozesses und aller Ebenen des komplexen Begriffs Medien annimmt bzw. zumindest begründet, warum eben nur einzelne Aspekte beleuchtet werden und sich der damit einher gehenden Lücken bewusst ist (vgl. Schmidt 2002; Jacke 2004).

Wie schwierig dieses Unterfangen ist, wird noch deutlicher, wenn man die Wechselwirkungen der einzelnen Ebenen bedenkt. Und hier stehen wir, wie eingangs warnend gesagt, erst ganz am Anfang der theoretischen Überlegungen geschweige denn empirischen Anwendungen. Eine zukünftige wissenschaftliche Medienkritik kann aber zumindest bei der klaren Zuordnung der Ebenen beginnen. Und sie muss sich, wie am Beispiel DSDS gut zu beobachten, der Unkritisierbarkeit neuer Medien-Formate bewusst sein, da diese die Inhalte nicht mehr von ihren Kontextualisierungen trennen lassen. Die Rezipienten hören im Fall von DSDS nicht mehr den Song an sich, sondern das Phänomen im Ganzen; das Format hat sich durch permanente Reflexivität und Medienverbünde unangreifbar gemacht bzw. die herkömmliche Kritik sogleich mit eingebaut. Und genau an diesem Punkt sollten zukünftige Kritiken ansetzen, damit sie weder in die Versuchung kommen,

19 Neben der hier als Startoperation zugrunde gelegten Unterscheidung wissenschaftlich/nicht-wissenschaftlich gibt es im Rahmen von Medienkritik als Medienweiterverarbeitung in Form von Kommunikationsprotokollen, Kondensationen, metatextuellen Beschreibungen, Bewertungen, Erklärungen und Transformationen zudem die Unterscheidungsmöglichkeiten ökonomisch abhängig/ unabhängig, professionell/nicht-professionell und medienspezifisch/medienübergreifend (vgl. Schmidt 2000: 155-175). 
launische Stammtischgespräche auf theoretisierendem Plateau fortzuführen, noch in die autologische $>$ Falle der Massenmedien laufen. ${ }^{20}$

Um auf die Problematik einer vermeintlich notwendigen Souveränität und Exklusivität aller Kritik, wie sie noch von den Kritischen Theoretikern geleistet werden konnte, im Zeitalter des durchmedialisierten Alltags allerdings kaum noch praktikabel erscheint, aufmerksam zu machen, verweise ich nochmals auf den Medienwissenschaftler Wolfgang Ernst und dessen Überlegungen zur Medienarchäologie: »Wenn - zusätzlich zum Radio - alle Sinnesorgane direkt medial adressiert werden können, gibt es tatsächlich kein Außen der Medien mehr« (Ernst 2002: 158). Ein prominenter Vertreter der Popmusik jenseits jeglicher Casting-Shows hat dies bereits in den Neunziger Jahren ganz ähnlich in seinen Tagebüchern festgestellt und daraus seine Form von Kritik abgeleitet: "I like to infiltrate the mechanics of a system by posing as one of them, then slowly start the rot from the inside of the empire (Cobain 2002: 100). Dass Cobain in seiner Verweigerung gegenüber der Medienindustrie erfolgreich gescheitert ist, dadurch bei seinen Fans noch mehr Authentizität und Individualität zugesprochen bekam und genau dies wieder zum Medienthema werden konnte, wurde bereits an anderer Stelle ausführlich dargelegt (vgl. Jacke 1998). ${ }^{21}$ Was im vorliegenden Beitrag aufgedeckt werden sollte, sind die Schwierigkeiten, die bisherige kritische Medientheorien in ihrer Anwendbarkeit auf Fernsehformate wie DSDS haben, sowie die Diskussion, warum alle diese Ansätze ihre weiterhin aktuelle Berechtigung im Rahmen einer neuen wissenschaftlichen Medienkritik besitzen und wie diese in einem eigenen, an Siegfried J. Schmidts soziokulturellen Konstruktivismus angelehnten Ansatz münden. Nur so kann eine theoretische Schieflage bei der Bewertung von Medieninhalten und -formaten vermieden werden, die sich ergibt, wenn mit theoretischen Kanonen auf mediale Spatzen geschossen wird - und umgekehrt. Auch ein Format wie DSDS kann auf allen Ebenen beobachtet und bewertet werden, aber einzig etwa die stimmliche Qualität der Teilnehmenden oder die Intertextualitätspotenziale im Sinne Ecos (2002) zu begutachten, erscheint im Rahmen medienkulturwissenschaftlicher Behandlung defizitär.

Kurzum bedeutet DSDS: Keiner darf gewinnen, aber einer muss gewinnen, letztlich sollen aber möglichst viele neue Prominente gewonnen werden, um Quote und Folgeeinnahmen zu erwirtschaften. Und kann dies den Castingshow-Produzenten im Rahmen des Privatrundfunks vonseiten einer

20 Besonders heraus gestellt wurde diese Problematik in den letzten Jahren von Pierre Bourdieu, Wolfgang Ernst, Peter Klier und Siegfried J. Schmidt.

21 Vgl. zu Stars und erfolgreichem Scheitern auch Jacke/Zurstiege 2003 sowie zum gelungenen Scheitern in der Kunst Branz 2005. 
Medienkulturwissenschaft vorgeworfen werden? DSDS ist schließlich nichts als ein Wettbewerb der Musik- und Medienindustrie um die Ware, aber kein qualitativer Musikwettbewerb, eher eine Wettbewerbssimulation, um nach den Regeln des künstlerischen Wettbewerbs die Ökonomieeffektivität zu steigern - und die Kritik wirbt fleißig mit.

\section{Literatur}

Adorno, Theodor W. (1992). Einleitung in die Musiksoziologie: 12 theoretische Vorlesungen. Frankfurt/M.: Suhrkamp (8. Aufl.; E: 1962).

Adorno, Theodor W. (2003). Eingriffe. Neun kritische Modelle. Sonderausgabe 40 Jahre Edition Suhrkamp. Frankfurt/M.: Suhrkamp (Sonderausgabe; E: 1963).

Behrens, Roger (1999). „Die Rückkehr der Kulturindustriethese als Dancefloorversion. Zur Dialektik materialistischer Pop- und Subkulturkritik.«In: Kritische Theorie und Poststrukturalismus. Theoretische Lockerungsübungen. Hg. v. jourfixe-initiative berlin. Hamburg: Argument, S. 53-62.

Behrens, Roger (2003). »Krise und Illusion. Zur Philosophie der Massenkultur.« In: Ders., Krise und Illusion. Beiträge zur kritischen Theorie der Massenkultur. Münster u.a.: LIT, S. 32-65.

Behrens, Roger (2004). Kulturindustrie. Bielefeld: Transcript.

Bolz, Norbert (2004). "Lust der Negation. Die Geburt der Kritischen Theorie aus dem Geist des Ressentiments. «In: Merkur. Deutsche Zeitschrift für europäisches Denken 58, H. 665/666, S. 754-761.

Branz, Manuela (2005). »Gelungenes Scheitern. Scheitern in der Postmoderne.«In: Im Zoo der Kunst I: Out Of Africa. Hg. v. Thomas Zaunschirm (= Kunstforum International Bd. 174). Ruppichteroth: Kunstforum-Bücherdienst, S. 262-267.

Brock, Bazon (1989). »Das Veraltete der traditionellen Ästhetik - der neuen nicht? Theodor W. Adornos Kampf gegen Oberpriester und Oberkellner eines Wissenschaftsideals. «In: Kunst und Philosophie. Hg. v. Florian Rötzer (= Kunstforum International Bd. 100). Ruppichteroth: Kunstforum-Bücherdienst, S. 236-241.

Cobain, Kurt (2002). Tagebücher. Köln: Kiepenheuer \& Witsch.

Diederichsen, Diedrich (1999). Der lange Weg nach Mitte. Der Sound und die Stadt. Köln: Kiepenheuer \& Witsch.

Eco, Umberto (2002). »Die Innovation im Seriellen.«In: Ders., Über Spiegel und andere Phänomene. München: DTV (7. Aufl.; E: 1983), S. 155-180.

Ernst, Wolfgang (2002). »Medienanatomie statt Kulturkritik. «In: Zerstreute Öffentlichkeiten. Zur Programmierung des Geheimnisses. Hg. v. Jürgen Fohrmann und Arno Orzessek. München: Wilhelm Fink, S. 143-160.

Göttlich, Udo (2003). »Kulturindustrie.«In: Handbuch Populäre Kultur. Begriffe, Theorien und Diskussionen. Hg. v. Hans-Otto Hügel. Stuttgart, Weimar: Metzler, S. $45-48$.

Grossberg, Lawrence (1998). »Replacing Popular Culture.«In: The Clubcultures Reader. Readings in Popular Cultural Studies. Hg. v. Steve Redhead. Oxford, Malden: Blackwell, S. 199-219.

Habermas, Jürgen (1990). Strukturwandel der Öffentlichkeit. Untersuchungen zu einer Kategorie der bürgerlichen Gesellschaft. Frankfurt/M.: Suhrkamp (E: 1962). 
Habermas, Jürgen (1995a). Theorie des kommunikativen Handelns. Band 1: Handlungsrationalität und gesellschaftliche Rationalisierung. Frankfurt/M.: Suhrkamp (E: 1981).

Habermas, Jürgen (1995b). Theorie des kommunikativen Handelns. Band 2: Zur Kritik der funktionalistischen Vernunft. Frankfurt/M.: Suhrkamp (E: 1981).

Hall, Stuart (1999a). "Die zwei Paradigmen der Cultural Studies.« In: Widerspenstige Kulturen. Cultural Studies als Herausforderung. Hg. v. Karl H. Hörning und Rainer Winter. Frankfurt/M.: Suhrkamp, S. 13-42.

Hall, Stuart (1999b). »Kodieren/Dekodieren.«In: Cultural Studies. Grundlagentexte zur Einführung. Hg. v. Roger Bromley, Udo Göttlich und Carsten Winter. Lüneburg: zu Klampen, S. 92-110.

Holert, Tom/Terkessidis, Mark (Hg.) (1996). Mainstream der Minderheiten. Pop in der Kontrollgesellschaft. Berlin, Amsterdam: Edition ID-Archiv.

Horkheimer, Max/Adorno, Theodor W. (2000). Dialektik der Aufklärung. Philosophische Fragmente. Frankfurt/M.: Fischer (12. Aufl.; E: 1944/47).

Jacke, Christoph (1998). »Millionenschwere Medienverweigerer: Die US-Rockband NIRVANA. «In: Neues zum Umgang mit Rock- und Popmusik. Hg. v. Helmut Rösing und Thomas Phleps (= Beiträge zur Popularmusikforschung 23). Karben: Coda, S. 7-30.

Jacke, Christoph (2001). "White Trash und Old School: Prominente und Stars als Aufmerksamkeitsattraktoren in der Werbung. «In: a/ effektive Kommunikation: Unterhaltung und Werbung. Hg. v. Siegfried J. Schmidt, Joachim Westerbarkey und Guido Zurstiege. Münster: LIT, S. 197-212.

Jacke, Christoph (2004). Medien(sub)kultur. Geschichten, Diskurse, Entwürfe. Bielefeld: Transcript.

Jacke, Christoph/Zurstiege, Guido (2003). »Vom erfolgreichen Scheitern.« In: Hinlenkung durch Ablenkung. Medienkultur und die Attraktivität des Verborgenen. Hg. v. Christoph Jacke und Guido Zurstiege. Münster u.a.: LIT, S. 97-106.

Jacke, Christoph/Zurstiege, Guido (2005). "Schöner Schrott: Werbe-Rauschen im Kultur-Programm. «In: Kulturschutt. Über das Recycling von Theorien und Kulturen. Hg. v. Christoph Jacke, Eva Kimminich und Siegfried J. Schmidt (in Vorb.).

Kellner, Douglas (1992). „Toward a Multiperspektival Cultural Studies. "In: The Centennial Review 36, Nr. 1, S. 5-41.

Kellner, Douglas (1995). Media Culture. Cultural Studies, Identity and Politics between the Modern and the Postmodern. London, New York: Routledge.

Kellner, Douglas (2003). Media Spectacle. London, New York: Routledge.

Kendall, Gavin/Wickham, Gary (2001). Understanding Culture. Cultural Studies, Order, Ordering. London u.a.: Sage.

Lash, Scott (1996). »Reflexivität und ihre Doppelungen: Struktur, Ästhetik und Gemeinschaft." In: Reflexive Modernisierung. Eine Kontroverse. Hg. v. Ulrich Beck, Anthony Giddens und Scott Lash. Frankfurt/M.: Suhrkamp, S. 195-286.

Maase, Kaspar (2003). »Massenkultur.« In: Handbuch Populäre Kultur. Begriffe, Theorien und Diskussionen. Hg. v. Hans-Otto Hügel. Stuttgart, Weimar: Metzler, S. 48-56.

Miss Kittin (2005). »Untitled.«In: Gendertronics. Der Körper in der elektronischen Musik. Hg. v. club transmediale und Meike Jansen. Frankfurt/M.: Suhrkamp, S. 17-18.

Münch, Richard (1998). »Kulturkritik und Medien - Kulturkommunikation.« In: Medien-Kulturkommunikation. Hg. v. Ulrich Saxer (= Publizistik-Sonderheft 2). Opladen: Westdeutscher Verlag, S. 55-66. 
Neumeister, Andreas (2002). Angela Davis löscht ihre Website. Listen, Refrains, Abbildungen. Frankfurt/M.: Suhrkamp.

Paetzel, Ulrich (2001). Kunst und Kulturindustrie bei Adorno und Habermas. Perspektiven kritischer Theorie. Wiesbaden: DUV.

Prokop, Dieter (1974): Massenkultur und Spontaneität. Zur veränderten Warenform der Massenkommunikation im Spätkapitalismus. Frankfurt/M.: Suhrkamp.

Prokop, Dieter (2003): Mit Adorno gegen Adorno. Negative Dialektik der Kulturindustrie. Hamburg: VSA.

Rosenberg, Bernard/White, David Manning (Hg.) (1957). Mass Culture. The Popular Arts in America. Glencoe: The Free Press.

Schmidt, Siegfried J. (1999). »Kultur als Programm. Zur Diskussion gestellt.« In: Kultur, Identität, Europa: Über die Schwierigkeiten und Möglichkeiten einer Konstruktion. Hg. v. Reinhold Viehoff und Rien T. Segers. Frankfurt/M.: Suhrkamp, S. 120-129.

Schmidt, Siegfried J. (2000). Kalte Faszination. Medien - Kultur - Wissenschaft in der Mediengesellschaft. Weilerswist: Velbrück.

Schmidt, Siegfried J. (2002). »Medienwissenschaft und Nachbardisziplinen. «In: Einführung in die Medienwissenschaft. Konzeptionen, Theorien, Methoden, Anwendungen. Hg. v. Gebhard Rusch. Wiesbaden: Westdeutscher Verlag, S. 53-68.

Schmidt, Siegfried J. (2004a). Unternehmenskultur: Die Grundlage für den wirtschaftlichen Erfolg von Unternehmen. Göttingen: Velbrück.

Schmidt, Siegfried J. (2004b). Zwiespältige Begierden. Aspekte der Medienkultur. Freiburg i. Br.: Rombach.

Strinati, Dominic (1995). An Introduction to the Theories of Popular Culture. London, New York: Routledge.

Terkessidis, Mark (2005). »Distanzierte Forscher und selbstreflexive Gegenstände. Zur Kritik der Cultural Studies in Deutschland.«In: Kulturschutt. Über das Recycling von Theorien und Kulturen. Hg. v. Christoph Jacke, Eva Kimminich und Siegfried J. Schmidt (in Vorbereitung).

Vogl, Joseph (2002). »Apokalypse als Topos der Medienkritik." In: Zerstreute Öffentlichkeiten. Zur Programmierung des Geheimnisses. Hg. v. Jürgen Fohrmann und Arno Orzessek. München: Fink, S. 133-141.

Weinzierl, Rupert (2000). Fight the Power! Eine Geheimgeschichte der Popkultur und die Formierung neuer Substreams. Wien: Passagen.

Wenzel, Harald (2001). Die Abenteuer der Kommunikation. Echtzeitmassenmedien und der Handlungsraum der Hochmoderne. Weilerswist: Velbrück.

Willis, Paul E. (1978). „Symbol und Realität. Zur gesellschaftlichen Bedeutung der Popmusik. «In: Ästhetik und Kommunikation. Beiträge zur politischen Erziehung 9, H. 31, S. 44-55. 


\begin{abstract}
This paper describes the current problems of media culture theories concerning postmodern phenomena of popular culture such as Deutschland sucht den Superstar (DSDS, the German version of Pop Idol). A re-reading of three groups of different approaches is done: Critical Theory (especially of the Frankfurt School), media cultural studies and German socio-cultural constructivism. Using the phenomenon of DSDS as an example, it is shown that these approaches have different potentials of scientific and theoretical criticism. While the Frankfurt School argues on an abstract level of social theory and tries to criticise modern societies as a whole, cultural studies concentrate on the political dimensions of case studies. This paper argues that socio-cultural constructivism (as understood by S. J. Schmidt and the author) - especially its precise definitions of media, communication and culture can help best to analyse the complexity of DSDS and to criticize all the aspects and effects of DSDS.
\end{abstract}

